\title{
Predicting Corresponding Region in a Third View Using Discrete Epipolar Lines
}

\author{
Hiroaki Natsumi $^{1}$, Akihiro Sugimoto ${ }^{2,3}$, and Yukiko Kenmochi ${ }^{3}$ \\ ${ }^{1}$ Chiba University, Japan \\ ${ }^{2}$ National Institute of Informatics, Japan \\ ${ }^{3}$ Université Paris-Est, LABINFO-IGM, UMR CNRS 8049, A2SI-ESIEE, France \\ sugimoto@nii.ac.jp, y.kenmochi@esiee.fr
}

\begin{abstract}
The discrete epipolar line, a discrete version of the epipolar line, is recently proposed to give geometric relationships between pixels in two different views so that we can directly deal with pixels in digital images. A method is then proposed to determine the discrete epipolar line providing that fully calibrated images are available. This paper deals with weakly calibrated digital images and proposes a method for determining the discrete epipolar line using only weakly calibrated images. This paper also deepens the work further, presenting a method for identifying the corresponding region in a third view from a given pair of corresponding pixels in two views.
\end{abstract}

\section{Introduction}

Understanding the geometry of corresponding primitives across different views that arise from the perspective projection of $3 \mathrm{D}$ objects is fundamental for applications such as 3D reconstruction from stereo or motion, object recognition, image synthesis, image coding. In particular, the relationships between different views of a point in space have been deeply investigated and sufficient knowledge about epipolar geometry, more generally multi-view geometry, is already well established 389.

In the framework of multi-view geometry studied so far, points in images are assumed to be directly handled. In other words, digitization of image points is not concerned at all. In reality, however, we cannot deal with points themselves in digital images because digital images involve some digitization process. The smallest unit in digital images is not a point but a pixel. Therefore, even if geometric features are perfectly detected, corresponding points across two views, for example, do not necessarily satisfy the epipolar constraint because of digitization of images 211]. This problem cannot be overcome without paying attentions to pixels as the smallest unit of images.

Conventionally, digitization errors are always treated together with observation errors, and how uncertainty arising from errors propagates into the estimation of geometrical information such as epipolar lines is statistically analyzed, providing that knowledge of error statistics are known [7/13. The employed statistical model for errors, however, is independent of the digitization scheme, and, moreover, the justification of the employed model is not sufficiently discussed.

D. Coeurjolly et al. (Eds.): DGCI 2008, LNCS 4992, pp. 470 481, 2008.

(C) Springer-Verlag Berlin Heidelberg 2008 


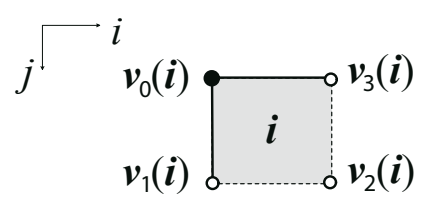

Fig. 1. The dominated region by pixel $i$ and its four corner vertices

Hamanaka et al. [5], on the other hand, proposed the discrete epipolar geometry, i.e., a discrete version of the conventional epipolar geometry where the pixel is highlighted as the smallest unit of images. The discrete epipolar geometry aims at rebuilding the conventionally known geometrical relationships on points between multiple views in order to directly deal with pixels in images. With its help, images with different resolutions can be handled simultaneously, for example. Reconstruction ambiguity caused by image digitization alone can be also clarified. However, the proposed method for determining from a given pixel, its discrete epipolar line, is not practically useful. This is because it assumes that images are fully calibrated in advance. This indicates that not only intrinsic camera parameters but also extrinsic ones are required to identify the discrete epipolar line for a given pixel.

This paper advances the direction of directly dealing with pixels, proposing a method for determining the discrete epipolar line using only weakly calibrated images. This paper also deepens the work further, presenting a method for identifying the corresponding region in a third view from a given pair of corresponding pixels in two views where images are assumed to be weakly calibrated. In the both methods, we move a pixel across two views with making full use of the fundamental matrix relating the two views. This allows us to obtain the discrete epipolar line and the corresponding region with only two-dimensional computation. To show the advantage of our approach, we demonstrate some experiments using images with different resolutions, which cannot be observed without paying attentions to the size of the smallest unit of images.

\section{Digitizing an Image into Pixels}

The smallest unit of digital images is not a point but a pixel. This indicates that an image is not continuous but digitized. We thus introduce a digitization to an uncalibrated image to obtain a set of pixels as its representation.

For a given point with homogeneous coordinates $\boldsymbol{x}=(x, y, 1)^{\top}$ in an uncalibrated image $\boldsymbol{I}$, we define $i=\left\lfloor r_{x} x+\frac{1}{2}\right\rfloor$ and $j=\left\lfloor r_{y} y+\frac{1}{2}\right\rfloor$, where $r_{x}, r_{y}$ are the resolutions of the $x$ - and $y$-coordinate, respectively. $\boldsymbol{i}=(i, j)^{\top}$ are the coordinates of the pixel representing $\boldsymbol{x}$. Applying this digitization to all the points in $\boldsymbol{I}$ leads to the digitization $\boldsymbol{D}$ of $\boldsymbol{I}$. We note that various digitization schemes exist to discuss geometric properties of digitized objects such as connectivities, bubble-freeness or topologies (see [110], for example); any digitization scheme causes no essential difference in subsequent discussion as far as any point in $\boldsymbol{I}$ corresponds to the unique pixel in $\boldsymbol{D}$. 


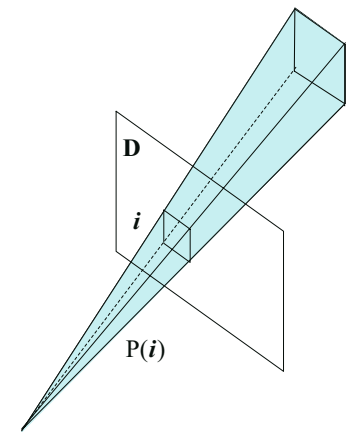

(a) pyramidal ray of sight

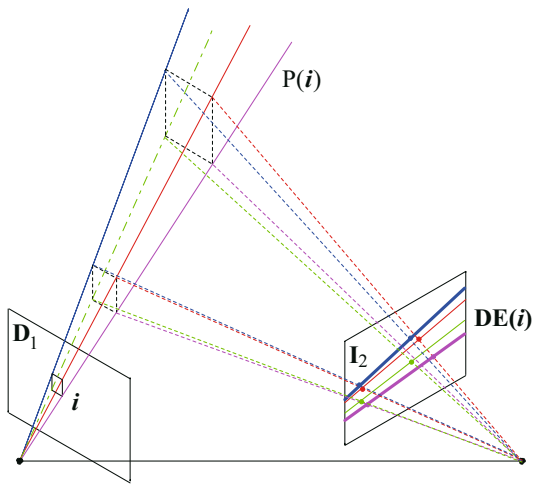

(b) discrete epipolar line

Fig. 2. Pixel-based geometry in the perspective projection

Conversely, the region dominated by a given pixel $i=(i, j)^{\top}$ is a rectangle and is easily computed. The four vertices determining the rectangle are called the corner vertices of $\boldsymbol{i}$ and denoted by $\boldsymbol{v}_{\kappa}(\boldsymbol{i}) \in \boldsymbol{I}(\kappa=0,1,2,3)$.

$$
\begin{aligned}
& \boldsymbol{v}_{0}(\boldsymbol{i})=\left(\frac{1}{r_{x}}\left(i-\frac{1}{2}\right), \frac{1}{r_{y}}\left(j-\frac{1}{2}\right), 1\right), \boldsymbol{v}_{1}(\boldsymbol{i})=\left(\frac{1}{r_{x}}\left(i-\frac{1}{2}\right), \frac{1}{r_{y}}\left(j+\frac{1}{2}\right), 1\right), \\
& \boldsymbol{v}_{2}(\boldsymbol{i})=\left(\frac{1}{r_{x}}\left(i+\frac{1}{2}\right), \frac{1}{r_{y}}\left(j+\frac{1}{2}\right), 1\right), \boldsymbol{v}_{3}(\boldsymbol{i})=\left(\frac{1}{r_{x}}\left(i+\frac{1}{2}\right), \frac{1}{r_{y}}\left(j-\frac{1}{2}\right), 1\right) .
\end{aligned}
$$

We note that only $\boldsymbol{v}_{0}(\boldsymbol{i})$ is included into the rectangle (Fig 10.

\section{Determining Discrete Epipolar Line}

\subsection{Discrete Epipolar Line}

The concept of discrete epipolar lines was introduced in [5]. The discrete epipolar line is geometrically defined as follows. A pixel in a calibrated image and the viewpoint define a quadrangular prism in space, called a pyramidal ray of sight for the pixel (Fig[2(a)). Projecting this pyramidal ray of sight onto the calibrated image observed from another viewpoint forms a discrete epipolar lin€ 1 (Fig $2(\mathrm{~b}))$.

As we can easily understand, the discrete epipolar line is bounded by two among four epipolar lines, each of which corresponds to one of the four corner vertices of the concerned pixel. The two epipolar lines that bound the discrete epipolar line are called bounding epipolar lines in this paper. Effectively identifying bounding epipolar lines under any viewpoint configuration thus becomes crucial in order to determine the discrete epipolar line.

\footnotetext{
${ }^{1}$ More precisely, the discrete epipolar line is the set of pixels intersecting with the image region of a pyramidal ray of sight. Namely, digitization of the image region in the second view is followed. The discrete epipolar line termed in this paper, however, is used without followed digitization. It is thus a continuous region represented by two inequalities. This causes no essential difference in discussion.
} 


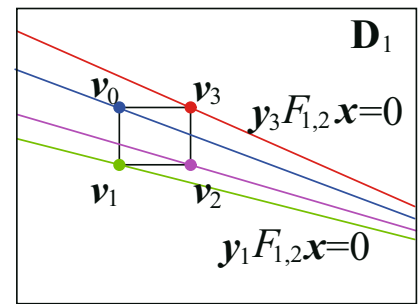

(a) 1st view

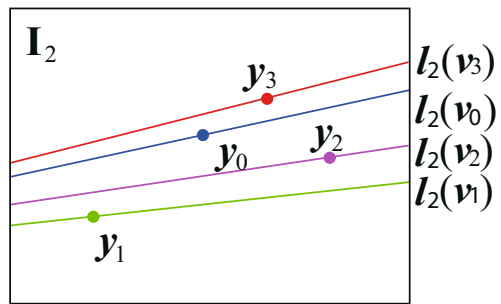

(b) 2nd view

Fig. 3. A given pixel and epipolar lines of its four corner points

Hamanaka et al. [5] proposed to use the epipolar planes of four corner vertices and selected two among the four so that the pyramidal ray of sight is fully included in between the selected epipolar planes. This is because the boundary of the discrete epipolar line is identical with the projection of the selected epipolar planes onto the second image. As images are assumed to be calibrated, however, not only intrinsic camera parameters but also extrinsic ones are explicitly required to identify the discrete epipolar line for a given pixel. This prevents discrete epipolar lines from their practical usefulness.

\subsection{Identifying the Boundaries Using the Fundamental Matrix}

A pair of corresponding points $\boldsymbol{x}_{1}, \boldsymbol{x}_{2}$ in two different uncalibrated images are known to be related by fundamental matrix $F_{1,2}$ :

$$
\boldsymbol{x}_{2}^{\top} F_{1,2} \boldsymbol{x}_{1}=0 \text {. }
$$

All the information about camera parameters is aggregated into $3 \times 3$ matrix $F_{1,2}$. Eq.(10) indicates that from a given point $\boldsymbol{x}_{1}$ in the first image, we can determine its corresponding epipolar line $\boldsymbol{x}_{1}^{\top} F_{1,2}^{\top} \boldsymbol{x}^{\prime}=0$ in the second image if two images are weakly calibrated, i.e., $F_{1,2}$ is known, where $\boldsymbol{x}^{\prime}$ is the homogeneous coordinates of a point in the second image. In the below, we assume that two images are weakly calibrated.

An epipolar line divides an image into two parts. The projection of the pyramidal ray of sight is included in only one part if the line is a bounding epipolar line, while it overlaps with two parts otherwise. This property allows us to discriminate bounding epipolar lines from others. We investigate this not in the second image but in the first image. This is because the given pixel is nothing but the projection of the pyramidal ray of sight onto the first image. We note that epipolar lines are pair-wisely determined in the first and second images.

Let $\boldsymbol{I}_{1}, \boldsymbol{I}_{2}$ be given two images, and $\boldsymbol{D}_{1}$ be the digitization of $\boldsymbol{I}_{1}$ (Fig, 3 ). For a given pixel $\boldsymbol{i} \in \boldsymbol{D}_{1}$, we have four corner points $\boldsymbol{v}_{\kappa}(\boldsymbol{i}) \in \boldsymbol{I}_{1}(\kappa=0,1,2,3)$, each of which determines epipolar line $\boldsymbol{\ell}_{2}\left(\boldsymbol{v}_{\kappa}(\boldsymbol{i})\right)$ in $\boldsymbol{I}_{2}$. We then select any point $\boldsymbol{y}_{\kappa}\left(\in \boldsymbol{I}_{2}\right)$ on the epipolar line $\boldsymbol{\ell}_{2}\left(\boldsymbol{v}_{\kappa}(\boldsymbol{i})\right)$. $\boldsymbol{y}_{\kappa}$ together with $F_{1,2}$ determines epipolar line $\boldsymbol{y}_{\kappa}^{\top} F_{1,2} \boldsymbol{x}=0$ in $\boldsymbol{I}_{1}$, where $\boldsymbol{x} \in \boldsymbol{I}_{1}$. We see that two epipolar lines $\boldsymbol{\ell}_{2}\left(\boldsymbol{v}_{\kappa}(\boldsymbol{i})\right)$ and $\boldsymbol{y}_{\kappa}^{\top} F_{1,2} \boldsymbol{x}=0$ are pair-wisely corresponding with each other, and, therefore, $\boldsymbol{y}_{\kappa}^{\top} F_{1,2} \boldsymbol{x}=0$ is independent of the choice of $\boldsymbol{y}_{\kappa}$. 
Once $\boldsymbol{y}_{\kappa}^{\top} F_{1,2} \boldsymbol{x}=0$ is obtained, it is easy to check the geometrical relationship between the region dominated by the given pixel $\boldsymbol{i}$ and two parts of $\boldsymbol{I}_{1}$ divided by $\boldsymbol{y}_{\kappa}^{\top} F_{1,2} \boldsymbol{x}=0$. Since $\boldsymbol{y}_{\kappa}^{\top} F_{1,2} \boldsymbol{x}=0$ always goes through the corner vertex $\boldsymbol{v}_{\kappa}(\boldsymbol{i})$, we have only to check the sign of $\boldsymbol{y}_{\kappa}^{\top} F_{1,2} \boldsymbol{v}_{\mu}(\boldsymbol{i})$ for the rest corner vertices $\boldsymbol{v}_{\mu}(\boldsymbol{i})(\mu=0,1,2,3 ; \mu \neq \kappa)$ to see whether or not the region dominated by $\boldsymbol{i}$ exists in only one part divided by $\boldsymbol{y}_{\kappa}^{\top} F_{1,2} \boldsymbol{x}=0$.

The algorithm below computes the discrete epipolar line from a given pixel. We note that $\boldsymbol{v}_{0}(\boldsymbol{i})$ is included into pixel $\boldsymbol{i}$; thus the case of $\kappa=0$ is distinctively treated.

Input: pixel $\boldsymbol{i}$ in the first image and fundamental matrix $F$ relating two images. Output: discrete epipolar line DE for $\boldsymbol{i}$ in the second image.

Step 1 Let $\mathrm{DE}:=\phi$.

Step 2 For $\kappa=0,1,2,3$, compute corner vertex $\boldsymbol{v}_{\kappa}(\boldsymbol{i})$ and epipolar line $\boldsymbol{\ell}_{2}\left(\boldsymbol{v}_{\kappa}(\boldsymbol{i})\right)$. Step 3 For $\kappa=0,1,2,3$, do

(1) let $W:=\{0,1,2,3\}-\{\kappa\}$;

(2) select a point $\boldsymbol{y}_{\kappa}$ on $\boldsymbol{\ell}_{2}\left(\boldsymbol{v}_{\kappa}(\boldsymbol{i})\right)$;

(3) if $\boldsymbol{y}_{\kappa}^{\top} F \boldsymbol{v}_{\mu} \geq 0$ for all $\mu \in W$, then $\left\{\right.$ if $\kappa=0$, then put $\boldsymbol{y}_{\kappa}^{\top} F \boldsymbol{x} \geq 0$ into DE; else put $\boldsymbol{y}_{\kappa}^{\top} F \boldsymbol{x}>0$ into DE $\}$;

(4) else if $\boldsymbol{y}_{\kappa}^{\top} F \boldsymbol{v}_{\mu} \leq 0$ for all $\mu \in W$, then $\left\{\right.$ if $\kappa=0$ then put $\boldsymbol{y}_{\kappa}^{\top} F \boldsymbol{x} \leq 0$ into DE; else put $\boldsymbol{y}_{\kappa}^{\top} F \boldsymbol{x}<0$ into DE $\}$.

\section{Predicting Corresponding Region in a Third View}

We assume that we are given two corresponding pixels in two different views. The problem addressed here is to determine the positions of corresponding pixels in a third view.

In the conventional framework, we can easily predict the position of the corresponding point in a third view from a given corresponding pair of points in two views. Namely, we first determine epipolar lines in a third view from a given pair of points in the first and second views, and then identify the intersection point of the two epipolar lines in the third view. We remark that the intersection point is always uniquely determined. This relationship between corresponding points across three views is algebraically analyzed and obtained constraints are called the trifocal tensor in the literature 4[6]12.

Once we accept the fact that the smallest unit of images is a pixel, however, the problem becomes hard to analytically solve, as seen below. We therefore algorithmically solve the problem.

\subsection{Corresponding Pixels in Two Views and a Third View}

When two corresponding pixels $\boldsymbol{i}_{1}, \boldsymbol{i}_{2}$ are given in two views, two pyramidal rays of sight in space are determined. We denote them by $P\left(\boldsymbol{i}_{1}\right)$ and $P\left(\boldsymbol{i}_{2}\right)$. The region in space that forms the given pixels as its image is identical with the intersection of the two pyramidal rays of sight, which is denoted by $P\left(\boldsymbol{i}_{1}\right) \cap P\left(\boldsymbol{i}_{2}\right)$. When a third viewpoint comes in, $P\left(\boldsymbol{i}_{1}\right) \cap P\left(\boldsymbol{i}_{2}\right)$ is projected onto the third view and forms a region in the third view. This region corresponds to $\boldsymbol{i}_{1}, \boldsymbol{i}_{2}$ and is denoted 
by $R_{3}\left(\boldsymbol{i}_{1}, \boldsymbol{i}_{2}\right)$. We call $R_{3}\left(\boldsymbol{i}_{1}, \boldsymbol{i}_{2}\right)$ the corresponding region for $\boldsymbol{i}_{1}$ and $\boldsymbol{i}_{2}$. As we easily see, $R_{3}\left(\boldsymbol{i}_{1}, \boldsymbol{i}_{2}\right)$ is not a point but a polygon.

The definition of a pyramidal ray of sight allows us to see that $P\left(\boldsymbol{i}_{1}\right)$ and $P\left(\boldsymbol{i}_{2}\right)$ have four faces and that they are a convex polyhedron. Accordingly, $P\left(\boldsymbol{i}_{1}\right) \cap P\left(\boldsymbol{i}_{2}\right)$ is a convex polyhedron with at most eight faces. Depending on not only the positions of given $\boldsymbol{i}_{1}$ and $\boldsymbol{i}_{2}$ in two views but also the geometrical configuration of two viewpoints, the number of faces of $P\left(\boldsymbol{i}_{1}\right) \cap P\left(\boldsymbol{i}_{2}\right)$ changes. This indicates that the shape of $R_{3}\left(\boldsymbol{i}_{1}, \boldsymbol{i}_{2}\right)$ itself is not invariant against these factors. This kind of changes does never arise in the conventional framework. In contrast, convexity is preserved against any changes in positions of $\boldsymbol{i}_{1}$ and $\boldsymbol{i}_{2}$, and in geometrical configuration of two viewpoints because the perspective projection does not break convexity. These observations can be summarized as follows.

For given two pixels in two views, the corresponding region in a third view

- is a convex polygon under any configuration of viewpoints and any positions of the given pixels;

- has at most eight edges, and the number of edges depends on the geometrical configuration of viewpoints and also on the positions of the given pixels.

\subsection{Identifying the Corresponding Region}

Straightforwardly using discrete epipolar lines to predict a corresponding region in a third view does not effectively work. This can be easily understood from the fact that if we determine in a third view two discrete epipolar lines for a given pair of pixels and then compute the intersection of the two discrete epipolar lines, the obtained region is with at most four edges; this contradicts the property of the corresponding region addressed in the previous section.

In fact, two pyramidal rays of sight for given pixels carve each other, and, therefore, the corresponding region becomes strictly smaller than the simple intersection of the two discrete epipolar lines. For a given pair of corresponding pixels $\boldsymbol{i}_{1}$ and $\boldsymbol{i}_{2}$ in two views, let $\mathbf{D E R}_{3}\left(\boldsymbol{i}_{1}\right)$ and $\mathbf{D E R}_{3}\left(\boldsymbol{i}_{2}\right)$ be the regions satisfying the discrete epipolar line in a third view for $\boldsymbol{i}_{1}$ and $\boldsymbol{i}_{2}$, respectively. Then, $R_{3}\left(\boldsymbol{i}_{1}, \boldsymbol{i}_{2}\right) \subseteq \operatorname{DER}_{3}\left(\boldsymbol{i}_{1}\right) \cap \operatorname{DER}_{3}\left(\boldsymbol{i}_{2}\right)$.

To see how two pyramidal rays of sight carve each other, we focus on each ridge line of a pyramidal ray of sight and investigate how it intersects with the faces of the other pyramidal ray of sight in order to obtain all the vertices of convex polyhedron $P\left(\boldsymbol{i}_{1}\right) \cap P\left(\boldsymbol{i}_{2}\right)$. Investigating this in space is complicated whereas the projection onto each view makes this analysis even simpler.

Let us focus on the investigation about a corner point $\boldsymbol{v}_{\kappa}\left(\boldsymbol{i}_{1}\right)$ and pyramidal ray of sight $P\left(\boldsymbol{i}_{2}\right)$ (Fig 4$)$. We consider epipolar line $\boldsymbol{\ell}_{2}\left(\boldsymbol{v}_{\kappa}\left(\boldsymbol{i}_{1}\right)\right.$ ) for $\boldsymbol{v}_{\kappa}\left(\boldsymbol{i}_{1}\right)$ in the second view. We note that $\boldsymbol{\ell}_{2}\left(\boldsymbol{v}_{\kappa}\left(\boldsymbol{i}_{1}\right)\right)$ is the projection of the line in space going through the first viewpoint and $\boldsymbol{v}_{\kappa}\left(\boldsymbol{i}_{1}\right)$. The fact that this line in space intersects with $P\left(\boldsymbol{i}_{2}\right)$ is identical with the fact that $\boldsymbol{\ell}_{2}\left(\boldsymbol{v}_{\kappa}\left(\boldsymbol{i}_{1}\right)\right)$ intersects with $\boldsymbol{i}_{2}$. Furthermore, if it intersects in space, the projection of intersection points in space is identical with the intersection points of $\boldsymbol{\ell}_{2}\left(\boldsymbol{v}_{\kappa}\left(\boldsymbol{i}_{1}\right)\right)$ and $\boldsymbol{i}_{2}$ in the second view. This allows us to identify the projection of intersection points in space onto the second view without directly dealing with $3 \mathrm{D}$ information.

Once we identify the projection of intersection points in space onto the second view, it is then straightforward to predict the position of the corresponding point 


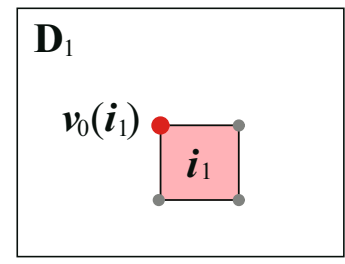

(a) 1st view

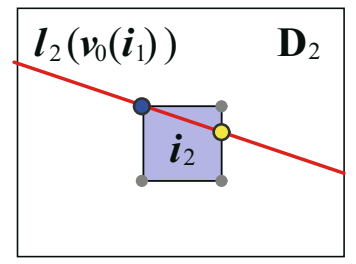

(b) 2nd view

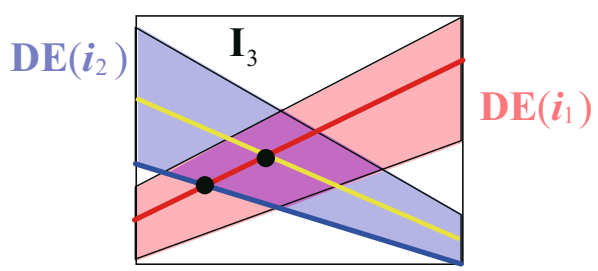

(c) 3rd view

Fig. 4. Intersection points of two pyramidal rays of sight observed in three views

in a third view. Namely, using fundamental matrices relating the third view with the first and second views, we determine epipolar lines in the third view for $\boldsymbol{v}_{\kappa}\left(\boldsymbol{i}_{1}\right)$ and also those for the obtained intersection points, and then compute intersection points to identify their positions in the third view.

Iterating the above procedure with respect to the four corner points $\boldsymbol{v}_{\kappa}\left(\boldsymbol{i}_{1}\right)$ ( $\kappa=0,1,2,3$ ) of $\boldsymbol{i}_{1}$ and then exchanging the roles of two views in the procedure lead to identifying the positions of the vertices in the third view, i.e., the projection onto the third view of all the vertices of $P\left(\boldsymbol{i}_{1}\right) \cap P\left(\boldsymbol{i}_{2}\right)$. Computing the convex hull of the obtained vertices in the third view enables us to identify the corresponding region $R_{3}\left(\boldsymbol{i}_{1}, \boldsymbol{i}_{2}\right)$. We remark that $R_{3}\left(\boldsymbol{i}_{1}, \boldsymbol{i}_{2}\right)$ is a convex polygon whose vertices are a subset of the obtained vertices.

The algorithm below identifies the corresponding region in a third view from given two corresponding pixels in two views.

Input: corresponding pixels $\boldsymbol{i}_{1}$ and $\boldsymbol{i}_{2}$ in the first and second views and fundamental matrices relating two of three views: $F_{1,2}, F_{1,3}, F_{2,3}$.

Output: corresponding region $R_{3}\left(\boldsymbol{i}_{1}, \boldsymbol{i}_{2}\right)$ in the third view.

Step 1. Let $F_{2,1}:=F_{1,2}^{\top}$ and $G:=\phi$.

Step 2. For $(\alpha, \beta) \in\{(1,2),(2,1)\}$, do

(1) for $\kappa=0,1,2,3$, do

(I) for $\tau=0,1,2,3$, do

(i) let $L_{\tau, \tau+1}^{\beta}$ bethelinesegment connecting $\boldsymbol{v}_{\tau}\left(\boldsymbol{i}_{\beta}\right)$ and $\boldsymbol{v}_{\tau+1(\bmod 4)}\left(\boldsymbol{i}_{\beta}\right)$;

(ii) compute in view $\beta$ the intersection point of line segment $L_{\tau, \tau+1}^{\beta}$ and epipolar line $\boldsymbol{v}_{\kappa}^{\top}\left(\boldsymbol{i}_{\alpha}\right) F_{\beta, \alpha} \boldsymbol{x}=0$; let the intersection point be $t$

(iii) compute the intersection of $\boldsymbol{v}_{\kappa}\left(\boldsymbol{i}_{\alpha}\right)^{\top} F_{\alpha, 3}^{\top} \boldsymbol{x}=0$ and $\boldsymbol{t}^{\top} F_{\beta, 3}^{\top} \boldsymbol{x}=0$; put the intersection point into $G$. 
Step 3. Compute the convex hull of $G$; let $R_{3}\left(\boldsymbol{i}_{1}, \boldsymbol{i}_{2}\right)$ be the region inside of the obtained convex hull.

\section{Experiments}

We demonstrate some experiments on predicting corresponding regions using weakly calibrated images with different resolutions. We remark that images with different resolutions are frequently obtained when they are captured using different digital cameras.

We set up experimental conditions as shown in Fig. [5 (a). Namely, for a randomly selected point $\boldsymbol{X}_{0}$ in space, we first put two viewpoints, $\boldsymbol{C}_{1}$ and $\boldsymbol{C}_{2}$, so that they together with $\boldsymbol{X}_{0}$ form the regular triangle in space, and obtained its two image points using camera parameters. We then put a third viewpoint $\boldsymbol{C}_{30}$ to form the regular tetrahedron together with $\boldsymbol{X}_{0}, \boldsymbol{C}_{1}$ and $\boldsymbol{C}_{2}$, and computed fundamental matrices determined by these views. After digitizing the first and second views, we applied our method to identify the corresponding region in the third view. We also conducted experiments in the cases where the third viewpoint is changed to $\boldsymbol{C}_{31}$ and $\boldsymbol{C}_{32}$ so that it becomes close to the first viewpoint $\boldsymbol{C}_{1}$ and the second viewpoint $\boldsymbol{C}_{2}$, respectively. This is to evaluate how the corresponding region depends on the viewpoint configuration in space. In our experiments, we fixed the resolution of the first view, and changed that of the second view to see how the corresponding region in the third view depends on resolutions. The resolution ratio of the second view to the first view was set to be $2^{k}(k=-3,-2,-1,0,1,2,3)$. Under the same condition except for changing $\boldsymbol{X}_{0}$ to $\boldsymbol{X}_{1}$ (Fig.[5] (b)), we conducted the same experiments.

The results for point $\boldsymbol{X}_{0}$ are shown in Fig. [6 while those for point $\boldsymbol{X}_{1}$ are in Fig.7. In these figures, (a), (c) and (e) illustrate the shape of the computed corresponding region, where the horizontal axis and the vertical axis correspond to the horizontal image coordinate and the vertical image coordinate, respectively. (b), (d), (f), on the other hand, show the number of edges and the area of the computed corresponding region, where the horizontal axis indicates the image resolution. We note that the vertical axis for the area of the computed corresponding region indicates the logarithm of the area to the base two.

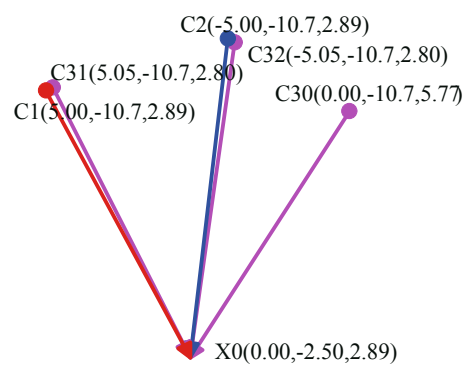

(a) case for a point $\boldsymbol{X}_{0}$

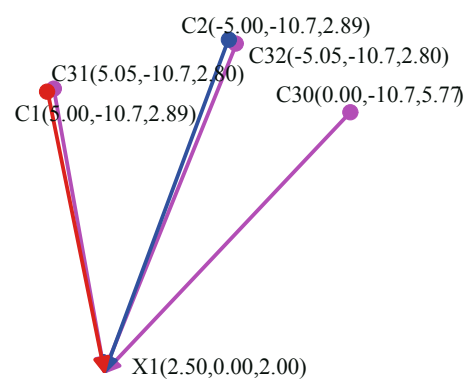

(b) case for a point $\boldsymbol{X}_{1}$

Fig. 5. Configuration of viewpoints $\boldsymbol{C}_{1}, \boldsymbol{C}_{2}, \boldsymbol{C}_{30}, \boldsymbol{C}_{31}$ and $\boldsymbol{C}_{32}$ 


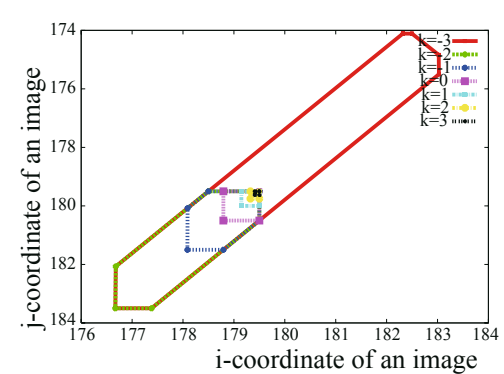

(a) shape (with $\boldsymbol{C}_{30}$ )

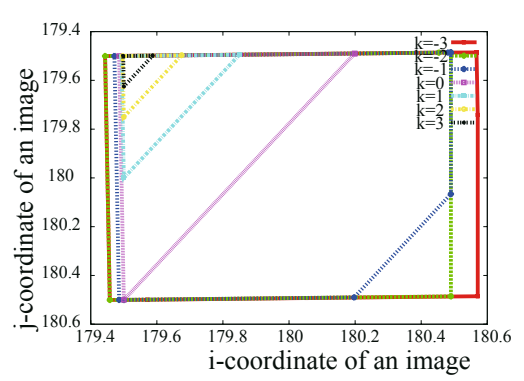

(c) shape (with $\boldsymbol{C}_{31}$ )

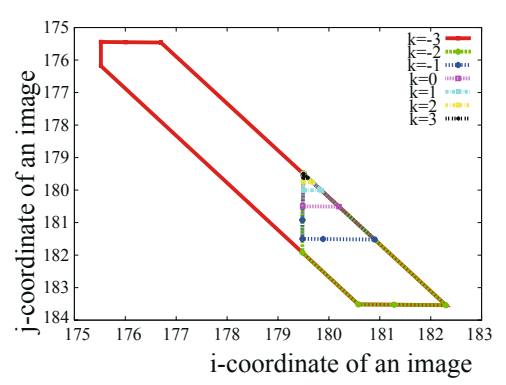

(e) shape (with $\boldsymbol{C}_{32}$ )

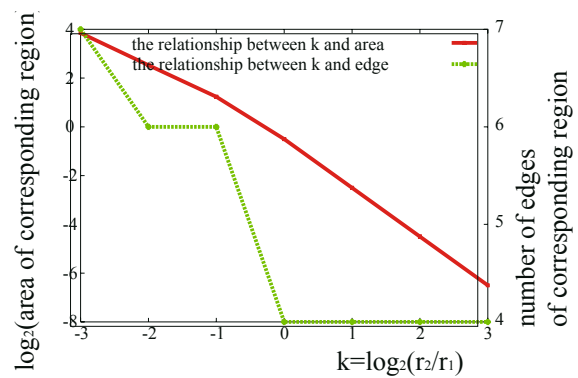

(b) edges and area (with $\boldsymbol{C}_{30}$ )

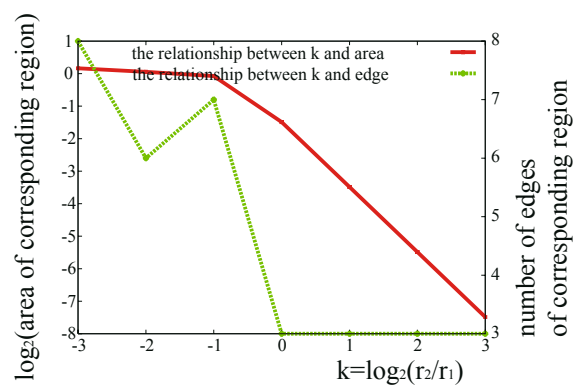

(d) edges and area (with $\boldsymbol{C}_{31}$ )

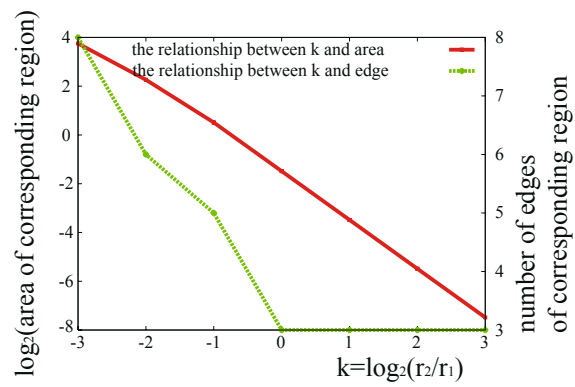

(f) edges and area (with $\boldsymbol{C}_{32}$ )

Fig. 6. Shape, edges and area of the corresponding region depending on image resolutions (case with viewpoints $\boldsymbol{C}_{1}, \boldsymbol{C}_{2}$ and point $\boldsymbol{X}_{0}$ )

Figures 6 and 7 show that in any case, as the resolution becomes higher the shape and the area of the computed corresponding region monotonically decrease. As we expect, the shape and the area tends to eventually converge to a point and zero, respectively. We also observe that the shape of the corresponding region itself changes depending on image resolutions even for the same point configuration. Furthermore, we observe some correlation exists between changes in number of edges and those in area; this observation is independent of the position of a third viewpoint. Namely, with a certain image resolution, both the area and the number of edges of the corresponding region significantly change at the same time and the changes depend on only the configuration of the first and the 


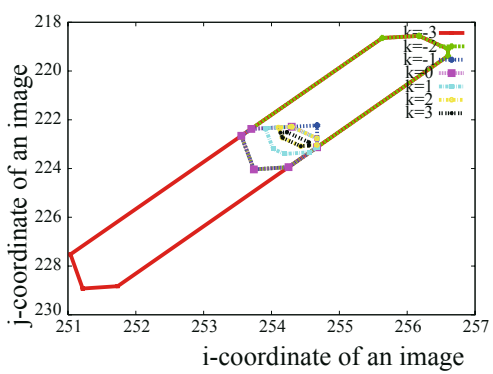

(a) shape (case with $\boldsymbol{C}_{30}$ )

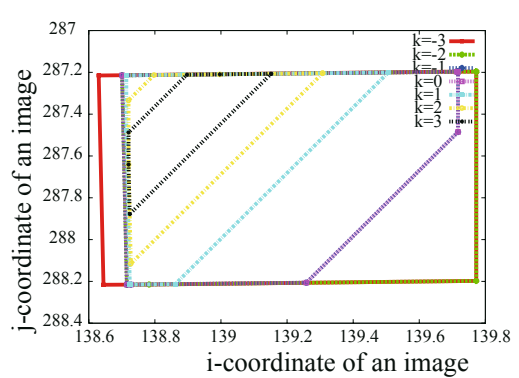

(c) shape (case with $\boldsymbol{C}_{31}$ )

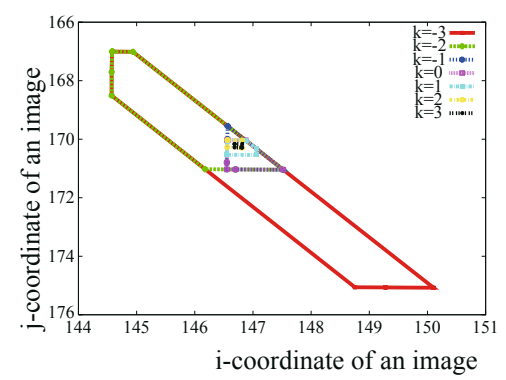

(e) shape (case with $\boldsymbol{C}_{32}$ )

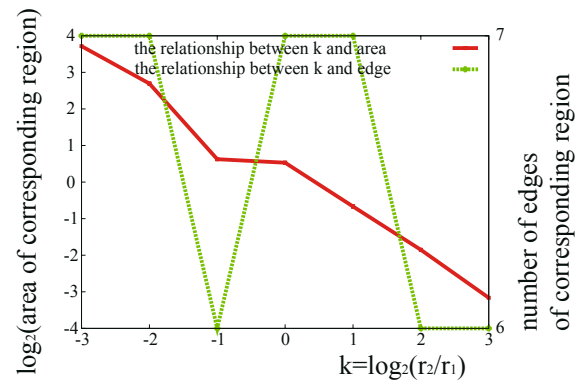

(b) edges and area (case with $\boldsymbol{C}_{30}$ )

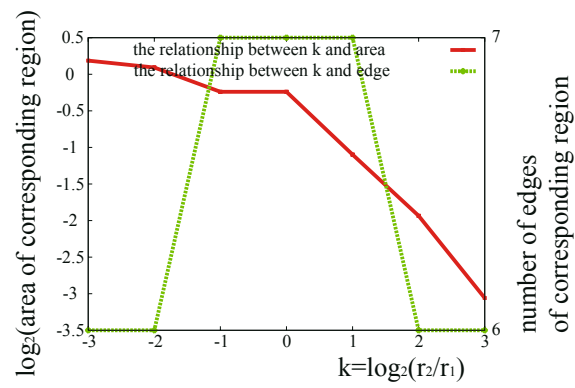

(d) edges and area (case with $\boldsymbol{C}_{31}$ )

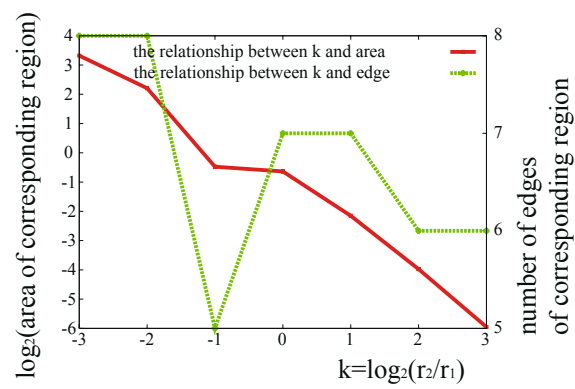

(f) edges and area (case with $\boldsymbol{C}_{32}$ )

Fig. 7. Shape, edges and area of the corresponding region depending on image resolutions (case with viewpoints $\boldsymbol{C}_{1}, \boldsymbol{C}_{2}$ and point $\boldsymbol{X}_{1}$ )

second viewpoints. These observations come from the fact that the intersection of two pyramidal rays of sight varies with image resolutions, and in particular, the shape of the intersection drastically changes with a certain image resolution.

Figure 6 (a), (b), (e) and (f) indicate the existence of the cases in which the area of the corresponding region has the same changes in shape depending on image resolutions though their viewpoint configurations are different from each other. This implies that when a third viewpoint is far from the first viewpoint, changes in resolution of the second view are irrelevant to the viewpoint configuration. This observation is also supported by Fig. 7 (a), (b), (e) and (f). 
On the other hand, we observe in (c) and (d) in Figs.6] and the existence of a critical resolution of the second view. Namely, when a third viewpoint is allocated closely to the first viewpoint, the area of the corresponding region remains almost invariant until the critical resolution, and once the second view achieves the critical resolution, the area then decreases significantly. This observation can be understood as follows. When the resolution of the second view is lower than that of the first view, the intersection of two pyramidal rays of sight is almost dominated by the pyramidal ray of sight of the first view, and, therefore, the image of the intersection is projected with the size of almost one pixel. This is because the image of the pyramidal ray of sight of the first view is exactly one pixel in the first view and because the third viewpoint is very close to the first viewpoint. As the resolution of the second view becomes higher than that of the first view, the intersection becomes gradually affected by the pyramidal ray of sight of the second view. The discrete epipolar line corresponding to the second view then chips in the third view the image of the pyramidal ray of sight of the first view to cause significant changes in area of the corresponding region.

The observations above are distinguished properties in dealing with pixels, and they cannot be obtained without paying attentions to the size of the smallest unit of images.

\section{Concluding Remarks}

This paper dealt with weakly calibrated images, focusing on pixels as the smallest unit of images. This paper then presented two methods: one for determining the discrete epipolar line from a given pixel, and the other for identifying the corresponding region in a third view from a given pair of corresponding pixels in two other views. Using fully calibrated images means that $3 \mathrm{D}$ information in the Euclidean sense is implicitly required while it is not in the case of weakly calibrated images. This indicates that our proposed methods do not require any three-dimensional computation at all; in fact, computation required here is just checking intersection between lines and points in images.

In the conventional approach, digitization errors and observation errors are not discriminated from each other and they are always treated together typically in the statistic framework. Our proposed methods, on the other hand, exactly identify how digitization errors propagate into the epipolar line estimation and how the ambiguity region looks like in a third image. Investigating practical superiority of our method to existing methods, in particular, the sub-pixel based method, is our next step. More detail analysis of the properties of the corresponding region from the theoretical point of view is also left for future work.

\section{References}

1. Andres, E.: Discrete Linear Objects in Dimension $n$ :the Standard Model. Graphical Models 65, 92-111 (2003)

2. Blostein, S.D., Huang, T.S.: Error Analysis in Stereo Determination of 3-D Point Positions. IEEE Trans. on PAMI 9(6), 752-765 (1987) 
3. Faugeras, O., Mourrain, B.B.: On the Geometry and Algebra of the Point and Line Correspondences between $N$ images. In: Proc. of ICCV, pp. 951-956 (1995)

4. Faugeras, O., Robert, L.: What can Two Images Tell Us about a Third One? IJCV 18(1), 5-19 (1996)

5. Hamanaka, M., Kenmochi, Y., Sugimoto, A.: Discrete Epipolar Geometry. In: Andrès, É., Damiand, G., Lienhardt, P. (eds.) DGCI 2005. LNCS, vol. 3429, pp. 323-334. Springer, Heidelberg (2005)

6. Hartley, R.: Lines and Points in Three Views and the Trifocal Tensor. IJCV 22(2), 125-140 (1997)

7. Hartley, R., Sturm, P.: Triangulation. CVIU 68(2), 146-157 (1997)

8. Hartley, R., Zisserman, A.: Multiple View Geometry in Computer Vision. Cambridge University Press, Cambridge (2000)

9. Heyden, A.: A Common Framework for Multiple View Tensors. In: Burkhardt, H., Neumann, B. (eds.) ECCV 1998. LNCS, vol. 1407, pp. 3-19. Springer, Heidelberg (1998)

10. Montanari, U.: On Limit Properties in Digitization Schemes. J. of ACM 17(2), 348-360 (1970)

11. Rodriguez, J.J., Aggarwal, J.K.: Stochastic Analysis of Stereo Quantization Error. IEEE Trans. on PAMI 12(5), 467-470 (1990)

12. Shashua, A., Werman, M.: Trilinearity of Three Perspective Views and its Associated Tensor. In: Proc. of ICCV, pp. 920-925 (1995)

13. Stewenius, H., Schaffalitzky, F., Nister, D.: How Hard is 3-View Triangulation Really? In: Proc. of ICCV, pp. 686-693 (2005) 